\title{
Angiographic detection of fatal acute aortic dissection Stanford type A under resuscitation
}

\author{
Jens Treptau ${ }^{1}$, Jens Ebnet ${ }^{2}$, Muharrem Akin ${ }^{1}$, Jörn Tongers ${ }^{1}$, \\ Johann Bauersachs ${ }^{1}$, Michael Brehm ${ }^{1}$, L. Christian Napp ${ }^{1}$ \\ ${ }^{1}$ Cardiac Arrest Center, Department of Cardiology and Angiology, \\ Hannover Medical School, Hannover, Germany \\ ${ }^{2}$ Department of Anaesthesiology, Hannover Medical School, Hannover, Germany
}

After resuscitation for out-of-hospital cardiac arrest (OHCA), emergent cardiac catheterization is recommended in patients with ST-segment elevation, and should be considered early in those without [1, 2]. Here, we present a patient with OHCA and inferior ST-segment elevation who inadvertently had fatal acute aortic dissection, which is only rarely documented by angiography.

A 77-year-old female with know atrial fibrillation on phenprocoumon and arterial hypertension was admitted to our emergency department after cardiopulmonary resuscitation (CPR) for OHCA. The patient had suffered from angina for $24 \mathrm{~h}$ and collapsed in the waiting room, while expecting an appointment with a general practitioner. After 20 min of CPR for asystole, spontaneous circulation returned. Electrocardiogram (ECG) demonstrated ST-segment elevation in leads II, III, aVF and V6. The ventilated patient, who was then stable with vasopressors, was immediately transferred to our hospital. After arrival in the emergency department, ECG confirmed ST-segment elevations, $\mathrm{Q}$ waves in leads II, III, aVF and V6, and incomplete right bundle branch block (Fig. 1A). Fast-track echocardiography showed mild aortic regurgitation but no pericardial effusion or severe right ventricular dysfunction. Therefore, the patient was transferred to the cathlab. On the way, hypotension occurred and CPR was performed with a mechanical resuscitation device (LUCAS, Physio-Control). Aortography revealed acute aortic dissection
Stanford type A (AADA) extending to both iliac arteries, with the brachiocephalic trunk and the left carotid artery originating from the false lumen (Fig. 1B, C; Supplementary Video 1 - see journal website). Due to prolonged resuscitation and fatal neurological prognosis, resuscitation was terminated. Autopsy confirmed AADA with an entry in the aortic bulb (Fig. 1D) without affecting the right coronary artery ostium, as well as an aneurysm of the abdominal aorta (Fig. 1E).

Prognosis of AADA is poor with $50 \%$ mortality after $48 \mathrm{~h}$ without surgery and $17.1 \%$ mortality after 30 days with surgery [3]. In our case, the clinical picture and ECG findings were indeed suggestive of inferior myocardial infarction, and typical echocardiographic signs of AADA, such as pericardial effusion or relevant aortic regurgitation were missing.

Incidence of AADA is low compared to that of ST-segment elevation myocardial infarction, but is probably underestimated [4]. The prevalence of ST-segment elevation in AADA is $3.2 \%$ [5], and fast rule-out echocardiography in patients with ST-segment elevation in inferior leads is useful [6] and allowed by current guidelines, as long as it does not delay angiography $[1,7]$. The present case prototypically illustrates fatal AADA and reminds us of considering AADA in cases of chest pain and ST-segment elevation in inferior leads, and further points to the eminent role of computed tomography in cases of successful resuscitation for OHCA [1].

Address for correspondence: L. Christian Napp, MD, Cardiac Arrest Center, Department of Cardiology and Angiology, Hannover Medical School, Carl-Neuberg-Str. 1, 30625 Hannover, Germany, tel: +49-511-532-2246, fax: +49-511-532-5412, e-mail: napp.christian@mh-hannover.de

Received: 15.09.2016 Accepted: 06.10.2016 


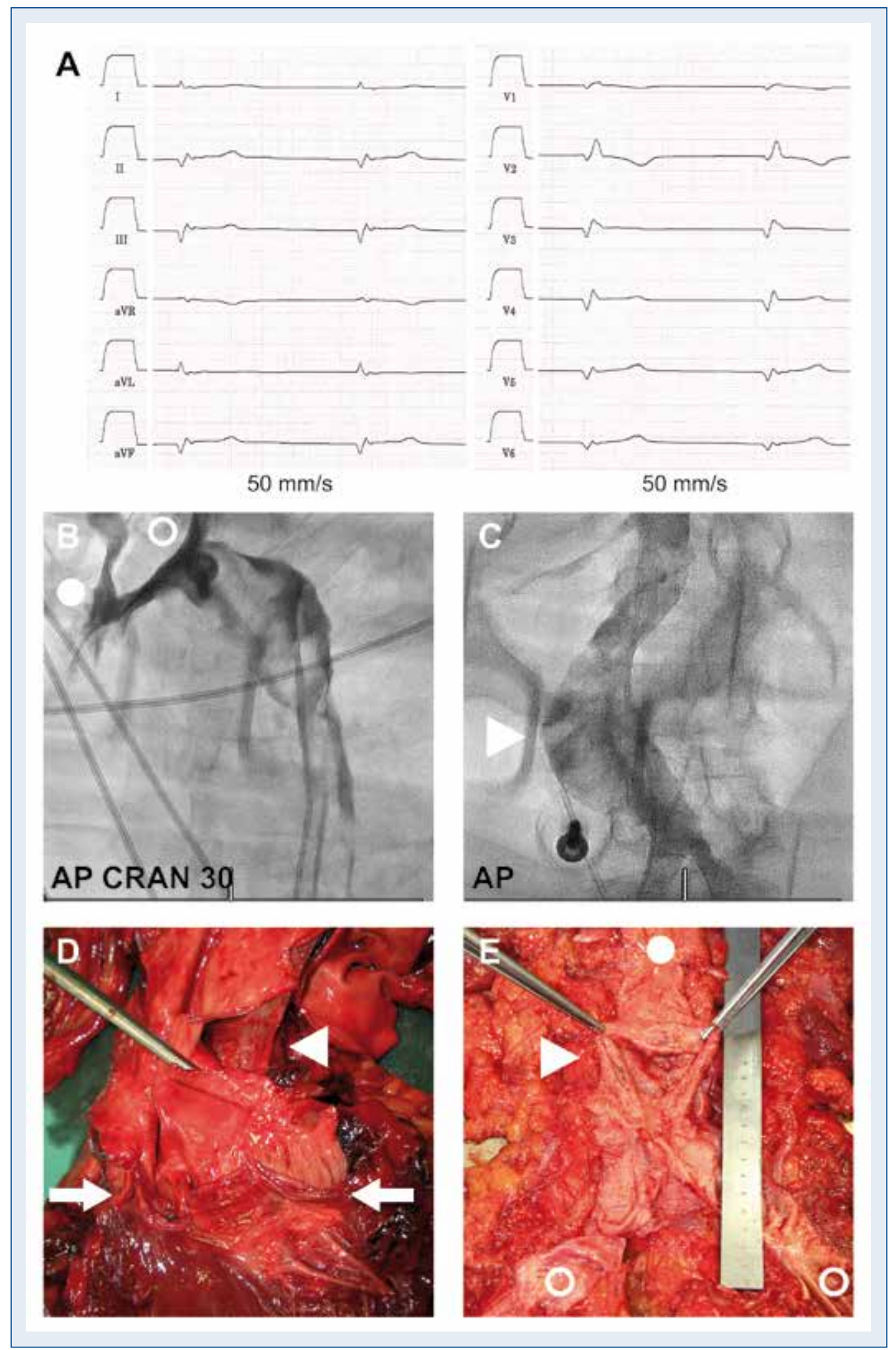

Figure 1. Electrocardiogram (ECG), angiography, and autopsy findings. A. ECG on admission demonstrating ST-segment-elevations and $\mathrm{Q}$ waves in II, III, aVF and V6; B. Angiography of the aortic arch with the pigtail-catheter in the false lumen, demonstrating the origin of brachiocephalic trunk (circle) and left carotid artery (open circle) from the false lumen; C. Angiography of the abdominal aorta aneurysm (arrowhead); D. Autopsy. Aortic bulb with dissection entry (arrowhead) proximal to the aortic valve (arrows); E. Autopsy. Abdominal aortic aneurysm (arrowhead) with disruption at its caudal part (circle: abdominal aorta, open circle: iliac artery). 


\section{Conflict of interest: None declared}

\section{References}

1. Nolan JP, Soar J, Cariou A et al. European Resuscitation Council and European Society of Intensive Care Medicine Guidelines for Post-resuscitation Care 2015: Section 5 of the European Resuscitation Council Guidelines for Resuscitation 2015. Resuscitation, 2015; 95: 202-222.

2. Noc M, Fajadet J, Lassen JF et al. Invasive coronary treatment strategies for out-of-hospital cardiac arrest: A consensus statement from the European association for percutaneous cardiovascular interventions (EAPCI)/stent for life (SFL) groups. EuroIntervention, 2014; 10: 31-37.

3. Rylski B, Hoffmann I, Beyersdorf F et al. Acute aortic dissection type A: Age-related management and outcomes reported in the German Registry for Acute Aortic Dissection Type A (GERAADA) of over 2000 patients. Ann Surg, 2014; 259: 598-604.

4. Clouse WD, Hallett JW, Jr., Schaff HV et al. Acute aortic dissection: Population-based incidence compared with degenerative aortic aneurysm rupture. Mayo Clin Proc, 2004; 79: 176-180.

5. Hagan PG, Nienaber CA, Isselbacher EM et al. The International Registry of Acute Aortic Dissection (IRAD): New insights into an old disease. JAMA, 2000; 283: 897-903.

6. Erbel R, Aboyans V, Boileau C et al. 2014 ESC Guidelines on the diagnosis and treatment of aortic diseases: Document covering acute and chronic aortic diseases of the thoracic and abdominal aorta of the adult. The Task Force for the Diagnosis and Treatment of Aortic Diseases of the European Society of Cardiology (ESC). Eur Heart J, 2014; 35: 2873-2926.

7. Steg PG, James SK, Atar D et al. ESC Guidelines for the management of acute myocardial infarction in patients presenting with ST-segment elevation. Eur Heart J, 2012; 33: 2569-2619. 